\title{
A new method for synthesis of hard dielectric coatings
}

\author{
Alexander S. Metel ${ }^{a}$ \\ Moscow State University of Technology "STANKIN", 1 Vadkovsky per., Moscow GSP-4, 127994, Russian Federation
}

Received 9 January 2017, Accepted 24 January 2017

\begin{abstract}
Aluminum oxide coatings have been synthesized on HSS, aluminum oxide and glass substrates using a magnetron sputtering system, which generates pulsed beams of high-energy gas atoms. The system comprises a flat grid positioned between the magnetron target and the substrates. Negative high-voltage pulses applied to the grid accelerate ions from the magnetron discharge plasma. Due to charge exchange collisions with gas molecules the ions turn into high-energy neutral atoms bombarding the coating growing on the substrate. They mix the substrate and coating atoms, thus enlarging the interface width, improving the coating adhesion and other properties.
\end{abstract}

Key words: Magnetron / sputtering / plasma / ions / charge exchange / fast atoms / pulsed beams / mixing / interface / adhesion / structure / modification

\section{Introduction}

For strengthening the surface of various industrial products, the ion-plasma treatment [1] is widely used. One of the strengthening methods is deposition of hard wear-resistant coatings, which substantially increase the useful life of the products. Metal atoms for synthesis of hard coatings are mainly produced using magnetron sputtering [2] or electron beam and vacuum arc evaporation [3,4]. When a pulsed dc magnetron [5] is used, sputtering rate of the target and plasma density near the substrate surface grow substantially. Another way to produce the metal atoms is sputtering of a target placed at the bottom of a hollow cathode $[6,7]$.

In all the above cases, the growing conductive coating is bombarded with ions accelerated from the discharge plasma by negative voltage of 50-200 V applied to a conductive substrate immersed in the plasma [3]. The ion bombardment improves the coating properties. To modify the properties with high-energy ions application to the substrate of high-voltage pulses is needed $[8,9]$. When to a HSS substrate are applied $20-\mu$ s-wide pulses with repetition frequency of $25 \mathrm{~Hz}$ and amplitude up to $50 \mathrm{keV}$, hardness of a magnetron sputtered TiN coating rises from 25 to $50 \mathrm{GPa}$ and the interface width exceeds $1 \mu \mathrm{m}$ [10]. It means that bombardment of the growing coating by high-energy particles allows production of superhard coatings $[11,12]$ and a substantial improvement of their adhesion.

\footnotetext{
${ }^{a}$ Corresponding author: a.metel@stankin.ru
}

It is impossible to apply high-voltage pulses to dielectrics. For this reason, the dielectric coating should be bombarded by fast neutral molecule beam. In known fast molecule beam sources $[13,14]$ the plasma emitter of ions is produced using glow discharge with electrostatic confinement of electrons [15], which is based on multiplication of fast electrons in the cathode sheath of the glow discharge $[16,17]$. The ions are accelerated by voltage between the plasma emitter filling a hollow cathode and secondary plasma in the chamber, both plasmas being separated from each other by a high transparency grid. Negative to the chamber potential of the grid (about 100$200 \mathrm{~V}$ ) prevents electrons of the secondary plasma from penetration into the plasma emitter. Ions accelerated in the positive space charge sheath between the plasma emitter and the grid enter the chamber through the grid orifices and on their way to the substrate turn into fast neutral molecules due to charge exchange collisions [18-20] with the gas molecules. Sputtering of a target placed opposite to the grid on the hollow cathode bottom results in a flow through the grid to the substrate of metal atoms accompanied by fast molecules [7]. Application of highvoltage pulses to the anode of hollow cathode glow discharge allows modification of growing coating by pulsed beams of high-energy gas atoms. Regrettably, due to electrical breakdowns, the above beam sources $[13,14]$ do not allow application of accelerating voltage exceeding $10 \mathrm{kV}$, and the highest energy of fast molecules so far reached amounts to $10 \mathrm{keV}$ [14].

It was shown in [21] that beams of fast neutral atoms and molecules can be produced due to charge exchange 
collisions of ions in the space charge sheaths near a negatively biased grid immersed in a low-pressure gasdischarge plasma. The plasma can be produced, for instance, by magnetron discharge. When we immerse in the discharge plasma between a flat magnetron target and a substrate a grid parallel to the target and apply to the grid negative high-voltage pulses, pulsed beams of high-energy gas atoms can be produced, which bombard the substrate during synthesis of a coating on its surface. Metal atoms, which take part in the synthesis, pass from the target to the substrate through the same grid. For this reason, their trajectories coincide with trajectories of high-energy gas atoms. Goal of the present work is synthesis of aluminum oxide coatings using such magnetron sputtering system generating pulsed beams of high-energy gas atoms.

\section{Experimental setup}

Figure 1 presents schematic of the experimental setup used for synthesis of dielectric coatings. Substrate 1 made of high-speed steel, aluminum oxide or glass is placed on holder 2 isolated from vacuum chamber 3 . Rectangular 13-cm-deep hollow case 4 is mounted on the chamber; its inner cross-section is equal to $60 \times 20 \mathrm{~cm}^{2}$. A planar magnetron is placed at the case bottom, its 15 -cm-wide and 37 -cm-long aluminum target 5 being cooled with water.

Connected between the target and grounded flange 6 power supply 7 ensures stabilized current of the magnetron discharge in the target circuit ranging from 1 to $8 \mathrm{~A}$ at the voltage up to $650 \mathrm{~V}$. Connected to the flange lateral plates 8 and 9 as well as the chamber and the case play the role of the discharge anode.

Inside the case on ceramic isolators (not shown in Fig. 1) is mounted grid 10. The isolators are protected from deposition on their surfaces of metallic films by anode plates 8 and 9. Distance between the grid and the inner surfaces of the case amounts to $1 \mathrm{~cm}$. It is possible to vary the distance between target 5 and grid 10 from 6 to $10 \mathrm{~cm}$. Using feed-through 11 the grid is connected to high-voltage pulse generator 12 . The generator allows regulation of the pulse amplitude from 3 to $30 \mathrm{kV}$, the pulse width from 5 to $50 \mu \mathrm{s}$, and the repetition rate from 5 to $50 \mathrm{~Hz}$. Holder 2 allows variation of the distance between substrate 1 and grid 10 from zero to $20 \mathrm{~cm}$.

After the magnetron discharge is ignited, the chamber is filled with glow of the discharge plasma, which is most intensive near the target surface (Fig. 2). Atoms 13 of the target material sputtered by ions 14 pass through the grid to the substrate and take part in the coating synthesis. When a high-voltage pulse is applied to grid 10, sheaths 15 and 16 of positive space charge appear near its surface and divide the discharge plasma into two parts 17 and 18. For this reason plasma 17, which fills the chamber, is not involved in the discharge during the pulse. In this time, only plasma 18 located between the target and the grid takes part in the ongoing magnetron discharge. Ions 19 are accelerated by the grid from plasma 18. Due to collisions in sheaths 15 and 16 with gas molecules 20 they

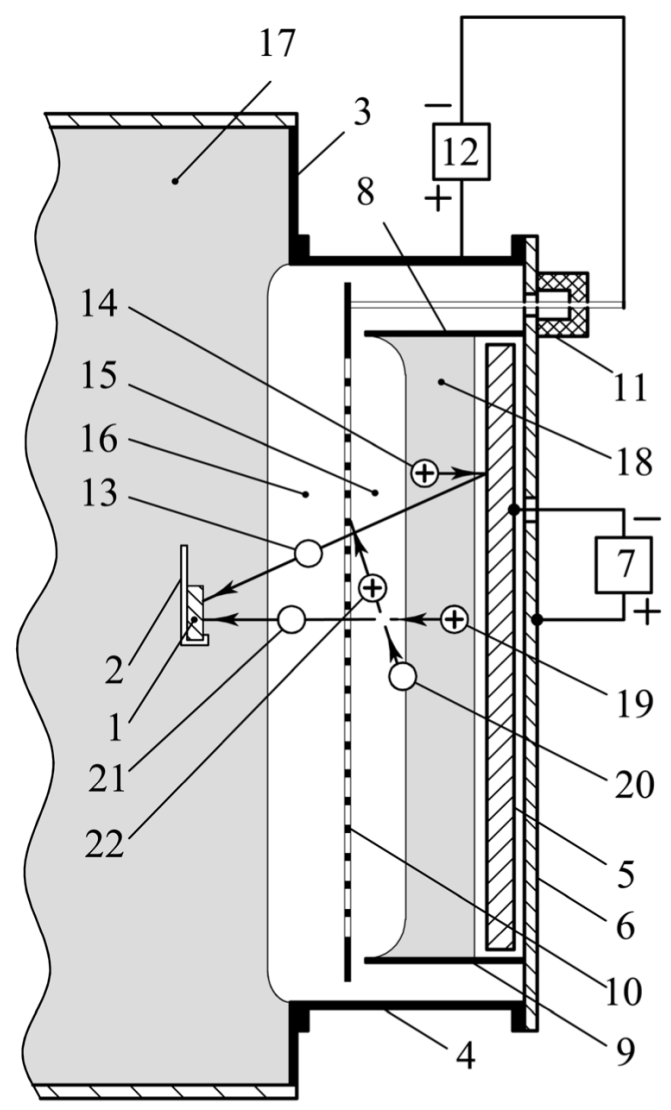

Fig. 1. Schematic of the experimental setup: (1) substrate; (2) holder; (3) chamber; (4) case; (5) target; (6) flange; (7) power supply; (8, 9) plates; (10) grid; (11) feed-through; (12) pulse generator; (13) target material atom; $(14,19,22)$ ions; $(15,16)$ space charge sheaths; $(17,18)$ plasma; $(20)$ gas molecule; (21) high-energy gas atom.

turn into fast atoms or molecules 21 bombarding substrate 1 . All ions 22 resulting from the charge exchange collisions are collected by grid 10 . Before placing the substrate on the holder, a 0.5 -mm-thick titanium mask is fixed on the substrate surface and they are cleaned in an ultrasonic bath.

After the coating deposition is finished, the mask is removed, and height of the step between the open surface of the substrate and its surface covered with the mask is measured using the DektakXT profilometer manufactured by Bruker Nano, Inc., USA (Fig. 3). The coating deposition rate is determined by dividing the height of the step by the deposition time.

\section{Experimental results}

When grid 10 (Fig. 1) with transparency of $70 \%$ is removed from its place, the deposition rate of aluminum coating at the current in the target circuit of $8 \mathrm{~A}$ and argon pressure of $0.5 \mathrm{~Pa}$ amounts to $2.5 \mu \mathrm{m} / \mathrm{h}$ at the distance from the target $h=20 \mathrm{~cm}, 9.5 \mu \mathrm{m} / \mathrm{h}$ at the distance $h=10 \mathrm{~cm}$ and $17 \mu \mathrm{m} / \mathrm{h}$ at the distance $h=5 \mathrm{~cm}$. After 


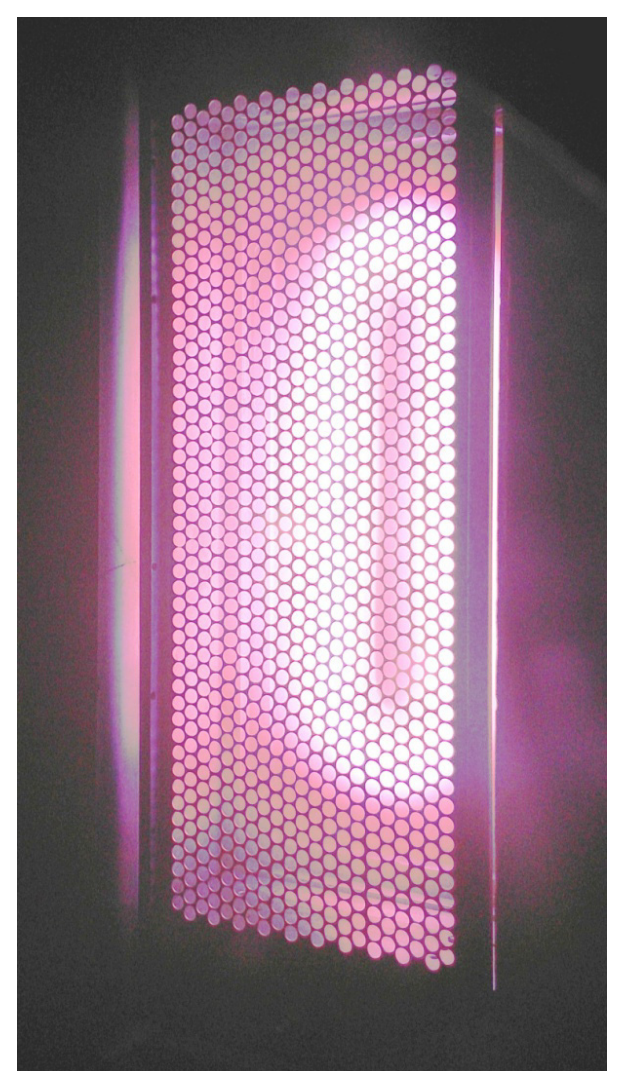

Fig. 2. Photograph of the discharge glow inside the chamber.

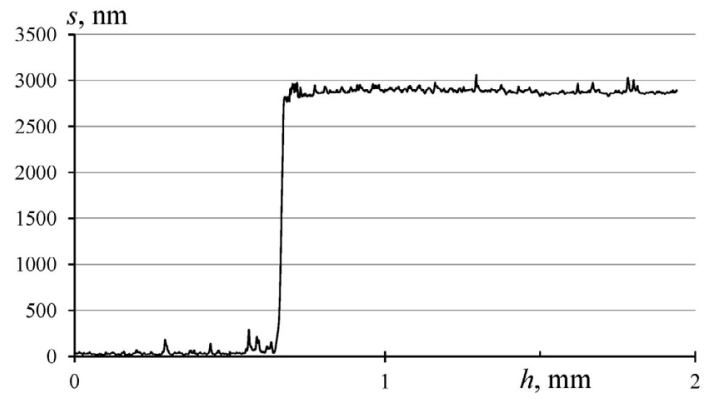

Fig. 3. Profilogram of a $3-\mu \mathrm{m}$-thick aluminum oxide coating synthesized at the target current of $6 \mathrm{~A}$ within $4 \mathrm{~h}$ on a HSS substrate distant at $3 \mathrm{~cm}$ from the grid.

the grid is reinstalled at his place and distanced from the target at $6 \mathrm{~cm}$, the aluminum coating deposition rate on the substrate distanced from the target at $9 \mathrm{~cm}$ diminishes at the same target current and argon pressure from 10 to $7 \mu \mathrm{m} / \mathrm{h}$, i.e. by $30 \%$, which is in good agreement with the grid transparency of $70 \%$.

Width of positive space charge sheath, which appear due to application to the grid of a high-voltage pulse, depends on the ion current density and the pulse amplitude. To determine the current density of ions 19 from plasma 18 (Fig. 1) located between grid 10 and target 5, dependence of the grid current $I_{\mathrm{g}}$ on the grid voltage $U_{\mathrm{g}}$ was obtained (Fig. 4). The current $I_{\mathrm{g}}$ first grows up with increasing the voltage $U_{\mathrm{g}}$, but at $U_{\mathrm{g}}$ amounting to hundreds of volts it abruptly diminishes by about 2 times and does

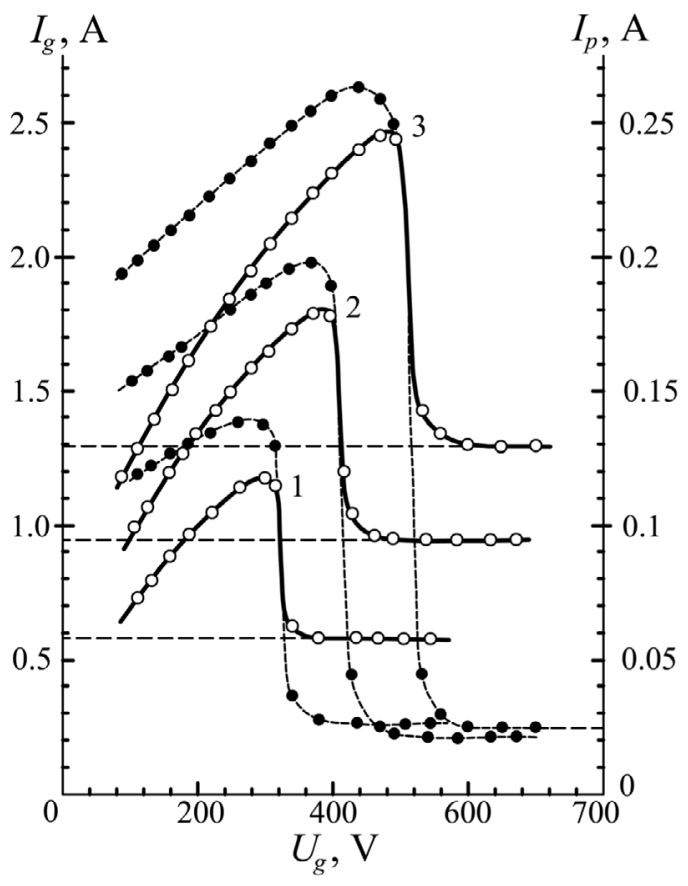

Fig. 4. Dependence on the grid voltage $U_{\mathrm{g}}$ of current $I_{\mathrm{g}}$ in its circuit at the distance $8 \mathrm{~cm}$ from the target (full curves) and ion current $I_{\mathrm{p}}$ in the circuit of a probe distant at $5 \mathrm{~cm}$ from the grid (dashed curves) at the target current 4 (1), 6 (2) and 8 A (3).

not change with further increasing the voltage. In this stage, intensity of the plasma glow inside the chamber falls down, and ion current in the circuit of a probe located in the chamber at the distance of $5 \mathrm{~cm}$ from the grid diminishes by an order of magnitude to a value, which is independent of the target current. In this situation, current in the grid circuit is approximately equal to the current $I_{\text {gi }}$ of argon ions accelerated from the plasma. At the distance $h=6 \mathrm{~cm}$ between the target and the grid it amounts to $2.3 \mathrm{~A}$ at the current in the target circuit of 8 A (Fig. 5), and the average ion current density on the grid surface amounts to $j=32 \mathrm{~A} / \mathrm{m}^{2}$.

When a $50-\mu$ s-wide high-voltage pulse is applied to the grid, the ion current in the grid circuit practically does not change, but the energy of arriving argon ions rises dramatically. At the pulse amplitude of $U=25 \mathrm{kV}$ the amplitude of current in the grid circuit reaches $9 \mathrm{~A}$. Increase of the current from 2.3 to $9 \mathrm{~A}$ is due to secondary electrons emission [22] induced by high-energy argon ions bombarding the grid.

For the above voltage and ion current density the Child-Langmuir law gives the sheath width value of $3.8 \mathrm{~cm}$. Argon ions from the magnetron discharge plasma are accelerated in the first sheath, enter the chamber through the grid orifices and are decelerated in the second sheath beyond the grid. Due to charge exchange collisions in the sheaths with gas molecules, they turn into highenergy neutral atoms. Energy of an argon atom corresponds to potential of the points, where the charge exchange collision took place. For this reason, the energy is 


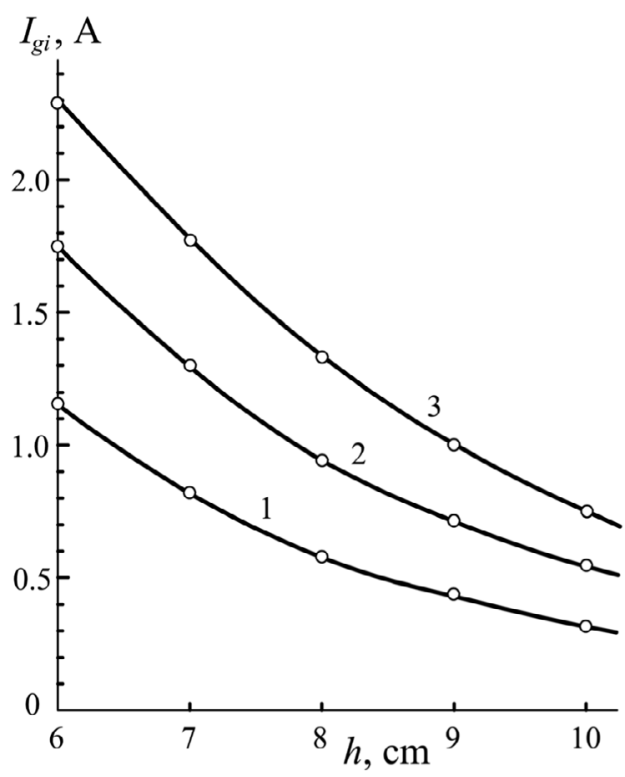

Fig. 5. Dependence on the distance $h$ between the target and the grid of the ion current $I_{\mathrm{gi}}$ in the grid circuit at the target current of 4 (1), 6 (2) and $8 \mathrm{~A}(3)$.

distributed continuously from zero to the maximal value of $25 \mathrm{keV}$.

Influence of high-energy gas atoms on the coating properties depends on the depth of their penetration into the substrate and coating materials, as well as on the ratio of the number of high-energy gas atoms implanted into the surface layer to the number of deposited atoms. The coating adhesion increases with growing flow of highenergy gas atoms into material as well as with growing depth of their penetration, which is directly proportional to their kinetic energy. In the present work evaluation of the fraction $N_{\delta} / N_{\mathrm{O}}$ of atoms with energy ranging from $\delta \varepsilon_{\mathrm{o}}$ to $\varepsilon_{\mathrm{o}}$ has been carried out, here $\delta<1, \varepsilon_{\mathrm{o}}=e U$ is the maximal energy of gas atoms, $N_{\delta}$ is their number, $U$ is the voltage applied to the grid and $N_{\mathrm{o}}$ is the number of ions entering the sheath from the discharge plasma adjoining the target.

The ratio $N_{\delta} / N_{\mathrm{o}}$ depends on the gas pressure. When the width of each sheath is equal to d, the ratio $N_{\delta} / N_{\mathrm{o}}$ reaches its maximal value at $d \approx \lambda$, here $\lambda=1 / n \sigma$ is the charge exchange length, $n$ is the gas molecule density and $\sigma$ is the charge exchange cross-section of argon ions, which at the ion energy ranging from 15 to $25 \mathrm{keV}$ may be assumed to be constant and equal to $\sigma=10^{-19} \mathrm{~m}^{2}$ [20]. At the gas pressure $1 \mathrm{~Pa}$ and room temperature $n=2.5 \times 10^{20} \mathrm{~m}^{-3}$, hence, the charge exchange length is equal to $\lambda=1 / n \sigma=0.04 \mathrm{~m}$. At the current $8 \mathrm{~A}$ in the target circuit, pulse amplitude of $25 \mathrm{kV}$, room temperature and argon pressure of $1 \mathrm{~Pa}$ the length $\lambda=4 \mathrm{~cm}$ is approximately equal to $d=3.8 \mathrm{~cm}$, and the number of gas atoms with energy ranging from 12.5 to $25 \mathrm{keV}(\delta=0.5)$ reaches its maximal value amounting to about $50 \%$ of all ions extracted from the plasma adjoining the target.
Experimental results proved that bombardment of growing coating by $25-\mathrm{keV}$ gas atoms allows synthesis of dielectric coatings with improved adhesion even without preliminary heating of the substrate and activation of its surface. When the magnetron discharge was switched on after application to the grid of high-voltage pulses, the substrate with temperature equal to the room temperature immediately found itself in the discharge plasma and the coating synthesis started. The substrate and the growing coating were bombarded by pulsed beams of highenergy gas atoms from the very beginning of the deposition processes. It was found that the coating deposition rate appreciably diminishes due to contamination of the aluminum target in oxygen. To compare the deposition rates at various oxygen percentage in the mixture with argon it was needed to create in each case the same starting conditions.

To this purpose before loading of each substrate into the chamber the aluminum target was cleaned during 20 $30 \mathrm{~min}$ at the magnetron discharge current of $8 \mathrm{~A}$ and pure argon pressure of $0.2 \mathrm{~Pa}$. Voltage of the discharge with contaminated target amounted to 200-250 V, but due to the target cleaning it increased to 350-400 V. The voltage increase indicated completion of the target cleaning.

At the voltage pulses amplitude $25 \mathrm{kV}$, width $40 \mu \mathrm{s}$ and frequency $50 \mathrm{~Hz}$, current in the target circuit of 6 $\mathrm{A}$ and argon pressure of $1 \mathrm{~Pa}$ the deposition rate of aluminum coating on a HSS substrate amounted to $5 \mu \mathrm{m} / \mathrm{h}$. At the same parameters addition to argon of $15 \%$ oxygen resulted in an only negligible decrease of the deposition rate and change of the coating white color to the gray color. The both coatings had the same phase constitution and specific conductance.

Further increase of oxygen percentage in mixture with argon to $18 \%$ resulted in dramatic changes of the process parameters and coating characteristics. The deposition rate diminished by an order of magnitude to $0.7 \mu \mathrm{m} / \mathrm{h}$, the coating became transparent and colorless, its specific conductance fell down to zero. At further increase of the oxygen percentage in the mixture with argon to $25 \%$ the deposition rate of the transparent dielectric coating smoothly diminished to $0.6 \mu \mathrm{m} / \mathrm{h}$. During $4 \mathrm{~h}$, a $3-\mu \mathrm{m}-$ thick transparent dielectric coating was produced on a steel substrate bombarded by $40-\mu$ s-wide pulsed beams of $15-\mathrm{keV}$ argon atoms with repetition rate of $50 \mathrm{~Hz}$ at the target current of $6 \mathrm{~A}$ and gas pressure of $1 \mathrm{~Pa}$. Using K-Alpha X-ray photoelectron spectrometer produced by Thermo Fisher Scientific company (Great Britain) the distributions of aluminum, oxygen and iron upon the depth of the coating and the substrate were obtained (Fig. 6).

They demonstrate a high uniformity of the coating composition and correspond to aluminum oxide (corundum). The distributions also made it possible to estimate the width of the intermediate layer between the substrate and the coating (interface), which amounts to $0.3 \mu \mathrm{m}$. When the amplitude of applied to the grid pulses rises to $25 \mathrm{kV}$, the interface width increases to $0.5 \mu \mathrm{m}$, when it 


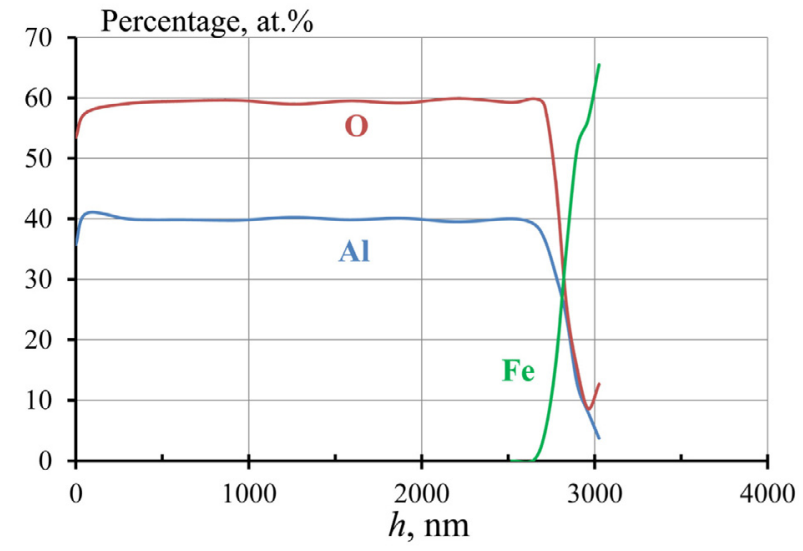

Fig. 6. Distributions of $\mathrm{Al}, \mathrm{O}$ and $\mathrm{Fe}$ atoms upon the depth of $3-\mu \mathrm{m}$-thick coating synthesized on a HSS substrate bombarded by $15-\mathrm{keV}$ argon atoms at the target current of $6 \mathrm{~A}$ and pressure $1 \mathrm{~Pa}$ of argon mixed with oxygen (20\%).

becomes lower than $5 \mathrm{kV}$, the interface width diminished to about $0.05 \mu \mathrm{m}$.

Using the scratch-tester by Nanovea company the first critical load was determined LC1 $=16 \mathrm{~N}$, at which first cracks appeared on the corundum coating bombarded during the synthesis on a high-speed steel substrate by argon atoms with maximal energy $25 \mathrm{keV}$. When the amplitude of pulses applied to the grid became lower than 5 $\mathrm{kV}$, the critical load diminished to $\mathrm{LC} 1=4 \mathrm{~N}$. Corundum coatings synthesized without application of the pulses to the grid exhibit the cracks even before the adhesion measurements. The same corundum coatings were also synthesized on substrates made of aluminum oxide and glass. Experiments showed that coating properties are practically independent of the substrate material.

\section{Discussions}

The data presented above confirmed that bombardment of growing coating by pulsed beams of high-energy gas atoms and molecules allows synthesis of hard dielectric coatings remarkable for good adhesion. Possibility to form the intermediate layer between the substrate and the coating (interface) at room temperature without any preheating and activation of the substrate is the most striking feature of coatings deposited using high-voltage pulses applied to the grid positioned between the magnetron target and the substrate. Measurements with a pyrometer showed that the substrate was heated to 200$300{ }^{\circ} \mathrm{C}$ by high-energy gas atoms and irradiation of the magnetron only in several minutes, when the interface formation was already completed. The use of a grid seems to be a simpler and more effective solution of the coating adhesion problem compared to pulsed dc magnetrons [5] or unbalanced magnetrons [23], because applied to the grid high-voltage pulses allow bombardment by energetic particles of dielectric substrates.

The adhesion dependence on the energy of gas atoms bombarding the substrate allows assumption of the sub- strate and coating atoms mixing by energetic atoms. Really, each of the gas atoms with energy of $25 \mathrm{keV}$ triggers dislodgement of about 500 atoms from the crystal lattices of the substrate and coating in the surface layer with a thickness of about $100 \mathrm{~nm}$. As a result, atoms of the substrate and the coating are mixed together. This is the reason of the interface width increase and the adhesion improvement. Depth of the gas atoms penetration into the substrate is proportional to their energy. Reduction of the high-voltage pulses amplitude should decrease the interface width.

It is confirmed by experimental results, which revealed the growth of the interface width from about 0.05 up to $0.5 \mu \mathrm{m}$ with increasing amplitude of applied to the grid pulses up to $25 \mathrm{kV}$ at the gas pressure of $1 \mathrm{~Pa}$. Deterioration of the adhesion with the pressure decrease from 1 to $0.2 \mathrm{~Pa}$ is due to the charge exchange length $\lambda$ increase. When $\lambda$ exceeds by several times the width $d$ of the space charge sheath near the grid surface, the number and energy of fast gas atoms bombarding the substrate also decrease.

\section{Conclusions}

1. Application of high-voltage pulses to a flat grid immersed in gas discharge plasma between a planar magnetron target and a substrate allows bombardment of the growing dielectric coating by pulsed beams of high-energy gas atoms. This enables synthesis of dielectric coatings with enhanced adhesion and hardness, for instance corundum coatings, on substrates made of conductive and dielectric materials.

2. Mixing of the substrate and dielectric coating atoms in the substrate surface layer due to bombardment of the substrate by high-energy gas atoms results in the coating adhesion improvement due to increase in the interface width.

Acknowledgements. This work was carried out with the financial support of the Ministry of Education and Science of Russian Federation in the framework of the state task in the field of scientific activity of MSTU "STANKIN" ( 519).

\section{References}

[1] S. Grigoriev, A. Metel, Plasma- and beam-assisted deposition methods, in: Nanostructured thin films and nanodispersion strengthened coatings. Kluwer Academic Publishers, Boston, Dordrecht, London (2004), pp. $147-154$

[2] J. Musil, A. Rajsky, A.J. Bell, J. Matous, M. Cepera, J. Zeman, High-rate magnetron sputtering, Journal of Vacuum Science and Technology A 14 (1996) 2187-2191

[3] R.L. Boxman, V.N. Zhitomirsky, Vacuum arc deposition devices, Review of Scientific Instruments 77 (2006) 021101/1-15 
[4] S.N. Grigoriev, A.S. Metel, S.V. Fedorov, Modification of the structure and properties of high-speed steel by combined vacuum-plasma treatment, Metal Science and Heat Treatment 54 (2012) 8-12

[5] J. Musil, J. Lestina, J. Vlcek, T. Tolg, Pulsed dc magnetron discharge for high-rate sputtering of thin films, Journal of Vacuum Science and Technology A 19 (2001) $420-424$

[6] S.N. Grigoriev, Yu.A. Melnik, A.S. Metel, V.V. Panin, V.V. Prudnikov, A compact vapor source of conductive target material sputtered by 3 -keV ions at $0.05-\mathrm{Pa}$ pressure, Instruments and experimental techniques 52 (2009) $731-737$

[7] A. Metel, V. Bolbukov, M. Volosova, S. Grigoriev, Yu Melnik, Source of metal atoms and fast gas molecules for coating deposition on complex shaped dielectric products, Surface and Coating Technology 225 (2013) 34-39

[8] A. Metel, Plasma immersion ion implantation based on glow discharge with electrostatic confinement of electrons, Surface and Coatings Technology 156 (2002) 38-43

[9] Handbook of plasma immersion ion implantation and deposition, A. Anders, editor, John Wiley \& Sons, Inc., New York (2000)

[10] C. Ruset, E. Grigore, The influence of ion implantation on the properties of titanium nitride layer deposited by magnetron sputtering, Surface and Coatings Technology 156 (2002) 159-161

[11] S. Veprek, H.-D. Mannling, P. Karvankova, J. Prochazka, The issue of the reproducibility of deposition of superhard nanocomposites with hardness of $\geqslant 50 \mathrm{GPa}$, Surface and Coating Technology 200 (2006) 3876-3885

[12] S. Veprek, Recent search for new superhard materials: Go nano!, Journal of Vacuum Science and Technology A 31(2013) 050822/1-33

[13] A.S. Metel, S.N. Grigoriev, Yu.A. Melnik, V.P. Bolbukov, Broad beam sources of fast molecules with segmented cold cathodes and emissive grids, Instruments and experimental techniques 55 (2012) 122-130

[14] A.F. Cakir, A. Metel, M. Urgen, S. Grigoriev, Arc-PVD coating of metallic and dielectric substrates using neutral molecular beam source pretreatment, Galvanotechnik 91 (2000) $768-776$

[15] V.I. Kolobov, A.S. Metel, Glow discharges with electrostatic confinement of fast electrons, Journal of Physics D: Applied Physics 48 (2015) 233001/1-19

[16] A.S. Metel, Effect of ionization in the cathode layer on the characteristics of a Penning discharge. I. Hollow cathode discharge, Soviet Physics - Technical Physics 30 (1985) $1133-1136$

[17] A.S. Metel, Characteristics of the detection of a quasistationary state of the heavy-current glow discharge with a hollow-space cathode at low gas pressure, Soviet Physics - Technical Physics 31 (1986) 1395-1405

[18] H.S.W. Massey, E.H.S. Burhop, Electronic and ionic impact phenomena, Clarendon Press, Oxford (1952)

[19] E.W. McDaniel, Collision phenomena in ionized gases, John Wiley \& Sons, Inc., New York, London, Sydney (1964)

[20] A.V. Phelps, Cross sections and swarm coefficients for nitrogen ions and neutrals in $\mathrm{N}_{2}$ and argon ions and neutrals in Ar for energies from $0.1 \mathrm{eV}$ to $10 \mathrm{keV}$, J. Phys. Chem. Ref. Data 20 (1991) 557-573

[21] A.S. Metel, Beams of fast neutral atoms and molecules in low-pressure gas-discharge plasma, Plasma Physics Reports 38 (2012) 254-262

[22] M. Kaminsky, Atomic and ionic impact phenomena on metal surfaces. Springer-Verlag, Berlin, Heidelberg, New York (1965)

[23] W.-D. Münz, F.J.M. Hauzer, D. Schulze, B. Buil, A new concept for physical vapor deposition coating combining the methods of arc evaporation and unbalancedmagnetron sputtering, Surface and Coating Technology 49 (1991) 161-167 